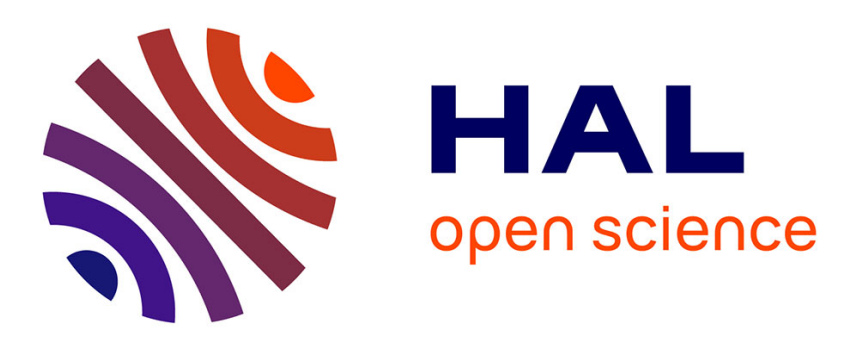

\title{
Electrical detection of internal dynamical properties of domain walls
}

\author{
Soraya Sangiao, Michel Viret
}

\section{To cite this version:}

Soraya Sangiao, Michel Viret. Electrical detection of internal dynamical properties of domain walls. Physical Review B: Condensed Matter and Materials Physics (1998-2015), 2014, 89, pp.104412. 10.1103/PhysRevB.89.104412 . cea-01377263

\section{HAL Id: cea-01377263 https://hal-cea.archives-ouvertes.fr/cea-01377263}

Submitted on 6 Oct 2016

HAL is a multi-disciplinary open access archive for the deposit and dissemination of scientific research documents, whether they are published or not. The documents may come from teaching and research institutions in France or abroad, or from public or private research centers.
L'archive ouverte pluridisciplinaire HAL, est destinée au dépôt et à la diffusion de documents scientifiques de niveau recherche, publiés ou non, émanant des établissements d'enseignement et de recherche français ou étrangers, des laboratoires publics ou privés. 


\title{
Electrical detection of internal dynamical properties of domain walls
}

\author{
Soraya Sangiao and Michel Viret ${ }^{*}$ \\ Service de Physique de l'Etat Condensé, CEA Saclay, DSM/IRAMIS/SPEC, bat 772, CNRS URA 2464, F-91191 Gif-sur-Yvette, France
}

(Received 29 October 2013; revised manuscript received 28 February 2014; published 13 March 2014)

\begin{abstract}
We report here on the electrical detection of the ferromagnetic resonance in a notched Py stripe using the spin rectification effect. A lock-in detection technique is used to measure the rectified voltage generated when applying a radio-frequency field. The multiple peaks associated with the presence of a domain wall, observed during field scans, are properly identified with the help of dynamical micromagnetic simulations. It is found that at any frequency below $6 \mathrm{GHz}$ some specific areas of the inhomogeneous magnetization within the domain wall can find a resonant condition and generate a voltage by rectification of the induction currents in the circuit.
\end{abstract}

DOI: $10.1103 /$ PhysRevB.89.104412

PACS number(s): 76.50.+g, 75.78.Cd, 75.78.Fg

\section{INTRODUCTION}

The new generation of electronic devices, so-called spintronic devices [1-3], combine standard electronics with spindependent effects and use the electron spin, rather than its charge, to carry information. The trend towards miniaturization in electronics has led to the need for understanding and controlling the magnetization dynamics of nanostructures. Present day lithographic techniques allow the patterning of magnetic nanostructures with controlled submicron size and shape. Classical inductive techniques do not reach the sensitivity allowing us to study the magnetization dynamics in individual magnetic nanostructures, but electrical transport has been shown to reach this goal $[4,5]$. Thus, the dynamics of the motion of domain walls (DWs) has been recently extensively studied both theoretically $[6,7]$ and experimentally $[4,8]$. It is typically found that DWs move at typical velocities of tens of metres per second corresponding to typical frequencies below $0.5 \mathrm{GHz}$ [9]. So far, most reported works concern the rigid motion of the walls within some artificially induced potential. The aim of the present paper is to detect the dynamics of the internal magnetic texture of a unique immobile DW. It corresponds to frequencies higher than $1 \mathrm{GHz}$, in the range of those used for usual ferromagnetic resonance (FMR).

For this purpose we fabricate $\mathrm{Ni}_{80} \mathrm{Fe}_{20}$ (Py) stripes with a notch along one of the edges that serves as an artificial pinning site for a DW. A micron sized stripline is also lithographied very close to the Py stripe in order to generate the excitation microwave field. We detect the DC voltage signal generated by rectification of the induction current in the conducting structure $[10,11]$. This voltage is caused by the spin-rectification effect $[12,13]$ that couples the induced rf current in the circuit containing the magnetic element to any change of resistance at the same frequency. In our samples the latter arises from the anisotropic magnetoresistance (AMR) that oscillates due to the precession of magnetization. Py stripes are attractive systems because they exhibit a sizable AMR contribution and have nearly zero magnetocrystalline anisotropy and magnetostriction, hence low damping. To further enhance the sensitivity of our measurements we modulate the rf field (at $\omega_{r f}$ ) by a square signal and measure the lock-in voltage generated across the stripe at the modulation

\footnotetext{
*michel.viret@cea.fr
}

frequency. The voltage picked up in our measurements arises from the current induced at $\omega_{r f}$ by the varying flux in the stripe rectified by mixing with the AMR contribution at $\omega_{r f}$. Thus, the line shape of the lock-in voltage we measure depends on the phase difference $\Psi$ between the rf magnetization and the rf induced current leading to a measured signal composed of a linear combination of symmetric and antisymmetric Lorentz line shapes [3].

\section{EXPERIMENT}

$10 \mu \mathrm{m}$ long, $1 \mu \mathrm{m}$ wide, and $20 \mathrm{~nm}$ thick Py stripes with a $600 \mathrm{~nm}$ wide notch are deposited on kapton substrates. The stripes are positioned with electron beam lithography $500 \mathrm{~nm}$ away from the current line of an $\mathrm{rf}$ antenna, previously defined by optical lithography (see Fig. 1). It is worth mentioning that we grow the samples on kapton, which is a flexible substrate, to use them also in break junction experiments, but kapton is not required to reproduce our results. The last step in sample fabrication consists in defining two $(\mathrm{Au})$ voltage contacts on each stripe, designed in a loop shape so that the induced current in the stripe is maximized. The sample is placed between the poles of a rotating electromagnet that provides DC magnetic fields up to $1.2 \mathrm{~T}$ at any in-plane angle. The voltage measurements are performed at room temperature, and an rf signal, square-wave modulated at $1.98 \mathrm{kHz}$, is sent to the antenna at frequencies up to $40 \mathrm{GHz}$ and at $0 \mathrm{dBm}$.

\section{RESULTS AND DISCUSSION}

We show in Fig. 2(b) the rectified voltage measured while sweeping the field at a constant frequency of $9 \mathrm{GHz}$, for different angles between the (in-plane) static magnetic field and the longitudinal axis of the stripe. A clear peak is measured at fields around $0.1 \mathrm{~T}$ for which the stripe is saturated, this is the FMR. Its exact position depends on the angle between the magnetization and the stripe direction (i.e., that of the induced current flow) but the line shape does not change. This is expected as, at a fixed frequency, the phase difference between the rf magnetization and the rf induced current remains unchanged. On the other hand, the voltage signal amplitude varies progressively and disappears at angles close to the transverse in-plane direction. In this configuration and for a $0.1 \mathrm{~T}$ field, the magnetization lays perpendicular to the stripe which makes the rectified voltage vanish [3]. Indeed, in 


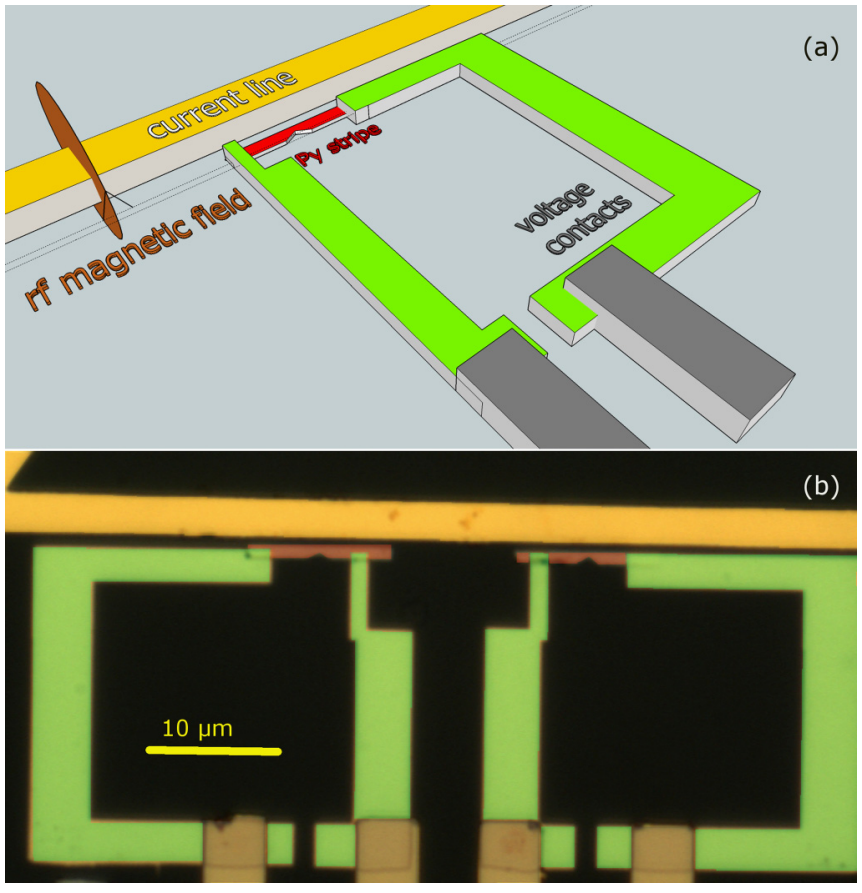

FIG. 1. (Color online) (a) Sketch of the samples designed for rectified voltage measurements. (b) (Artificially colored) optical image of a sample containing two Py stripes.

such a configuration, the AMR gives a signal at twice the rf frequency which cannot be rectified with the induced current. The measured peaks are therefore induced by the uniform FMR mode of the stripe.

Interestingly, when the frequency is lowered, a signal starts to appear near zero field as the sample is no longer homogeneously magnetized. This is shown in Fig. 3(a) for frequencies between 1 and $4 \mathrm{GHz}$ where it can be seen that the signal is the highest for $3 \mathrm{GHz}$. Moreover, the sample's resistance is also measured using a small current at $7 \mathrm{~Hz}$ driven through the sample while measuring the voltage at the same frequency. Figure 3(b) shows the sample resistive variation with the magnetic field applied at 90 degrees with respect to the stripe axis. The curve is globally bell shaped as the AMR progressively decreases when the angle between the magnetization and the stripe increases towards 90 degrees. A slight opening is also visible, both at positive and negative low fields, between approximately 10 and $15 \mathrm{mT}$. Interestingly, $10 \mathrm{mT}$ corresponds to the field at which a peak is observed in the rectified voltage coming back from positive saturation (and symmetrically $-10 \mathrm{mT}$ when coming from negative saturation). Such a curve opening in the magnetoresistance often indicates the appearance of an inhomogeneous magnetic state. In the particular case of our structure with an almost transverse applied field, it corresponds to the nucleation of a domain wall in the notch area $[14,15]$. The presence of the DW increases the total stripe resistance by around $0.6 \Omega$. For this reason, hereinafter we call this peak the nucleation peak. As visible in Fig. 3(b), the rectified voltage is much richer with the presence of several peaks in the field region where a DW is pinned at the notch. It is instructive to compare the rectified voltage to the field derivative of the

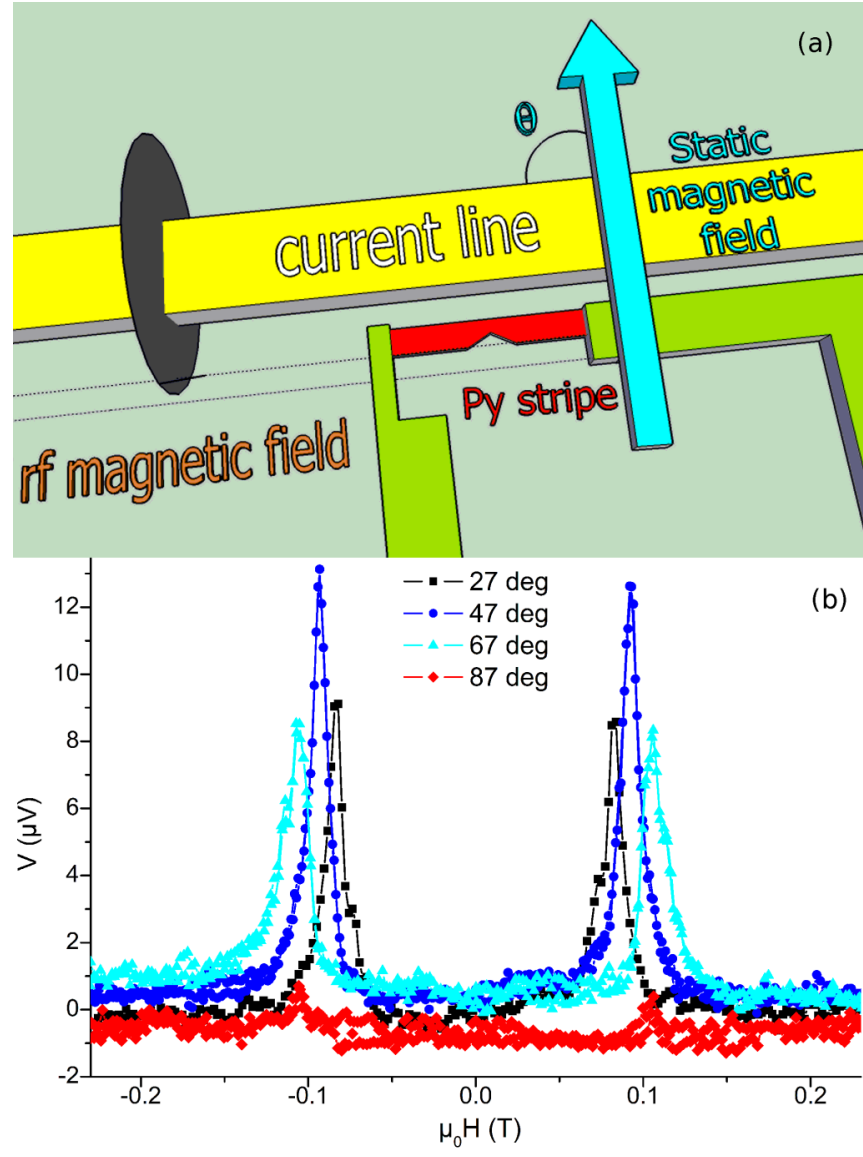

FIG. 2. (Color online) (a) Sketch of the measurement geometry. (b) Field dependence of the measured rectified voltage at $9 \mathrm{GHz}$ and $0 \mathrm{dBm}$ for different angles between the static magnetic field and the longitudinal axis of the stripe.

resistivity, a quantity equivalent to a resistive susceptibility near zero frequency. Indeed, it corresponds to the rectified voltage one would measure at low frequency in the presence of a constant current. Globally, their shapes are rather similar, but the rectified voltage presents extra features including a peak at $-35 \mathrm{mT}$ as well as the nucleation peak at $10 \mathrm{mT}$. It is also important to notice that the "saturation" peak at $-12 \mathrm{mT}$ appears before the actual saturation given by the peak in the $\mathrm{dR} / \mathrm{dH}$ curve. It is hard to understand the exact origin of these extra features but it is obvious that most of them appear when the magnetization is inhomogeneous, i.e., when a DW is present. Experimentally, it is also important to note that these extra peaks are only found for frequencies below $6 \mathrm{GHz}$. We believe this indicates that for these lower frequencies, the varying internal field near the notch provides regions in space where a resonant condition is met. In the following, we concentrate on the rectified voltage measurements performed at $3 \mathrm{GHz}$, at angles close to the transverse direction, which are representative of the DW effects on the RF susceptibility.

Figure 4(a) presents the measured rectified voltages at different applied angles in a range of 75 to 105 degrees from the stripe axis. The shape of the signal can be well understood considering that the rectified voltage is proportional to $\sin (\alpha) \cos (\alpha)$ with $\alpha$ the angle of the magnetization with 

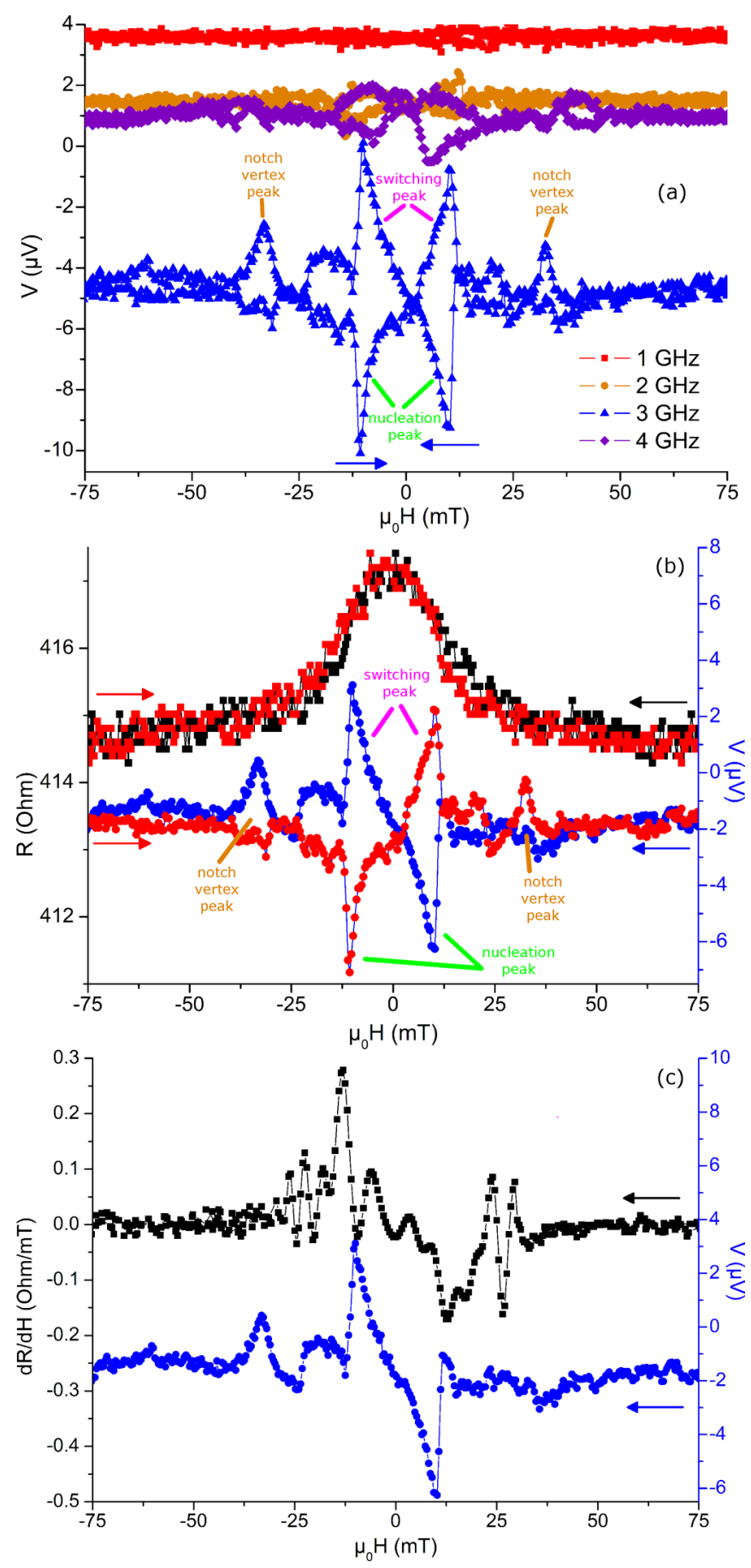

FIG. 3. (Color online) (a) Field dependence of the measured rectified voltage at an angle of 90 degrees between the static magnetic field and the longitudinal axis of the stripe for different frequencies up to $4 \mathrm{GHz}$ (the measurement at $3 \mathrm{GHz}$ has been shifted downwards by $-3 \mu \mathrm{V}$ for the sake of clarity). (b) Measurement of the total stripe resistance (black or red squares, left axis) and the rectified voltage (blue or red dots, right axis) at $3 \mathrm{GHz}$ as a function of the static magnetic field applied at an angle of 90 degrees with respect to the axis of the stripe. (c) Comparison of the rectified signal with the DC susceptibility $\mathrm{dR} / \mathrm{dH}$ extracted from the $\mathrm{R}(\mathrm{H})$ curve.

respect to the stripe. Hence, peaks signs are reversed when the magnetization angle goes from slightly below 90 degrees to above it. Moreover, near zero field, the signal sign depends
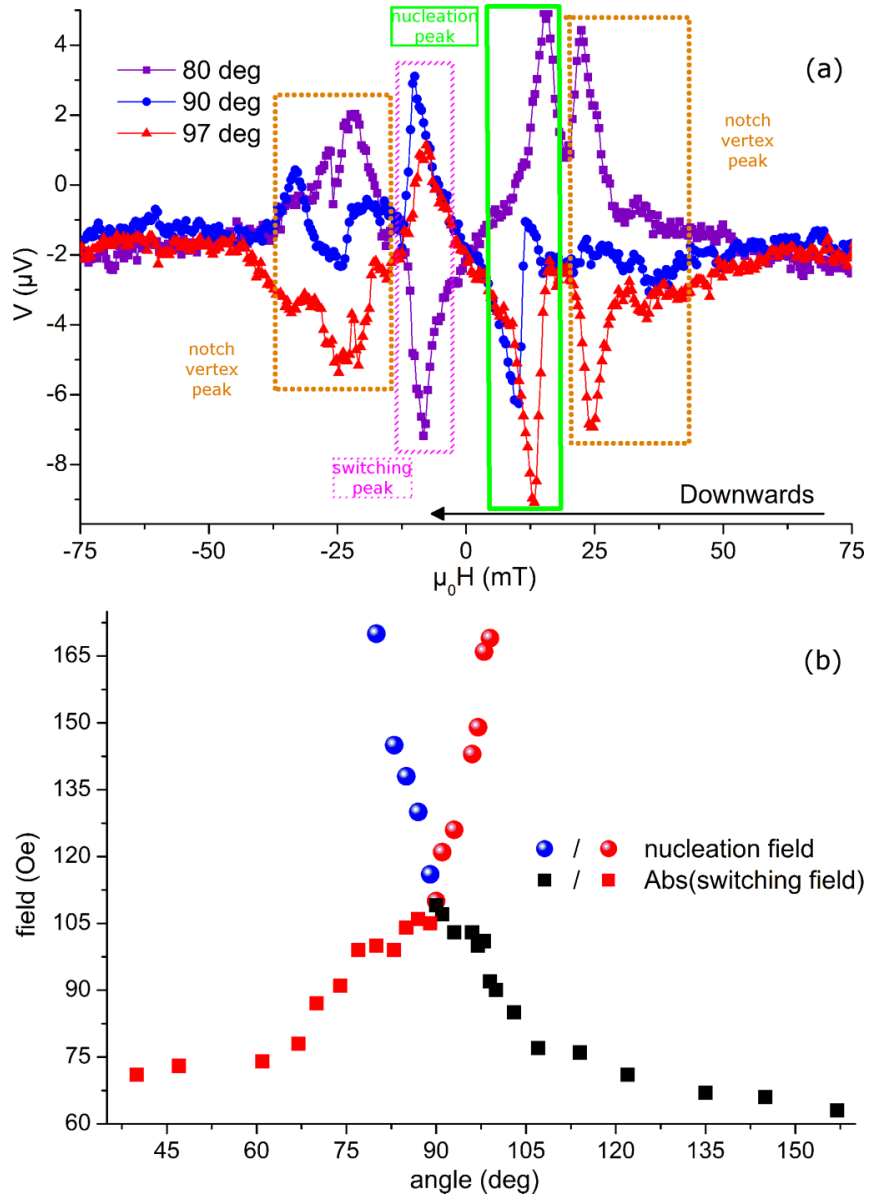

FIG. 4. (Color online) (a) Field dependence of the measured rectified voltage at $3 \mathrm{GHz}$ for different angles around the transverse direction. (b) Angular dependence of the nucleation and the switching fields (the switching field, negative, is represented in absolute value for the sake of clarity) at $3 \mathrm{GHz}$. Blue or black (red) symbols mean positive (negative) peak.

on the component of longitudinal magnetization, which is different when coming from positive or negative saturation fields. Nevertheless, apart from their signs, rather similar features are indeed observed in all these curves. Going from positive to negative fields, three different peaks can be seen:

(i) The notch vertex peak corresponding to the excitation of the precession in the area around the tip of the notch. It is the only peak we observe at both negative and positive fields (for example, at +25 and $-25 \mathrm{mT}$ when applying the magnetic field at 97 degrees with respect to the stripe axis).

(ii) The nucleation peak, when a DW starts to nucleate in the notch area.

(iii) The switching peak that happens just before the DW is depinned from the notch triggering magnetization reversal. This feature is actually not a real peak but should rather be viewed as a steep increase of the rectified signal completely suppressed as soon as the DW escapes the notch area.

The notch vertex excitation and the nucleation peaks are positive below 90 degrees and negative at angles above this value, and the opposite dependence is found for the switching peak, which is negative (positive) below (above) 90 degrees 
[see Fig. 4(a)], all these sign changes being consistent with the expected symmetry. As can be seen in Fig. 4(b), the switching field, in absolute value, is maximum at 90 degrees whereas the nucleation field is minimum at this angle.

The only peak that remains when measuring at angles not close to the transverse direction is the switching peak that can be observed at angles up (down) to 165 (15) degrees. This feature was previously reported by Grollier et al. [16], when the longitudinal magnetization reverses. In the longitudinal field geometry, the magnetization reversal does not take place by depinning of the pinned DW but rather by the nucleation of a reversed domain and subsequent DW propagation. In our measurements shown in Fig. 4(b), the nucleation field strongly increases when moving away from the transverse direction. A detailed study of its angular dependence would help us to gain a deeper insight into the precise mechanism of magnetization reversal, but this is not the aim of the present paper.

In order to understand in more detail the extra features in the rectified voltage, micromagnetic simulations were carried out using the MuMax2 code [17], which is a micromagnetic simulation tool running on graphical processing units. The simulations are performed in a $10 \times 1 \times 0.02 \mu \mathrm{m}^{3}$ volume with $20 \times 8 \times 5 \mathrm{~nm}^{3}$ unit cells with the exact shape of our Py stripe with a notch. The Py parameters are set to: saturation magnetization $\mathrm{M}_{S}=800 \mathrm{kA} \mathrm{m}{ }^{-1}$, exchange constant $\mathrm{A}=$ $1.3 \times 10^{-11} \mathrm{~J} \mathrm{~m}^{-1}$, and Gilbert damping parameter $\alpha=0.01$. We have followed a field scan procedure starting from positive transverse saturation and reducing the field. Each step uses as the initial state the magnetic configuration previously determined. The excitation frequency of the rf magnetic field has been chosen to be $f=3.0 \mathrm{GHz}$. The magnetic configurations obtained at different field values while sweeping the field, applied at an angle of 90 degrees, are shown on the left side of Fig. 5. The chosen field values correspond to the magnetic fields at which we observe each of the different peaks in our measurement [see Figs. 3(a) or 4(a)]: the nucleation peak at around $10 \mathrm{mT}$ [Fig. 5(a)], the switching peak at $-10.4 \mathrm{mT}$ [Figs. 5(c) and 5(d)], and the notch vertex excitation peak at $-32.5 \mathrm{mT}$ [Fig. 5(e)]. We have also included the magnetization configuration at zero field [Fig. 5(b.1)] in order to show the vortex wall pinned at the notch.

On the right side of Fig. 5 we display in black the sample outline and in color the areas in which the magnetization is found to oscillate for an rf excitation of $3 \mathrm{GHz}$. Several interesting features can be pointed out from this figure:

(i) As discussed above, the peak we measure around $+10 \mathrm{mT}$ corresponds to the nucleation of a DW at the notch area and, as can be seen in Fig. 5(a.2), the only region that oscillates at $+10 \mathrm{mT}$ is the center of the DW. We measure a well-defined peak in the rectified voltage at this magnetic field value, in spite of the small oscillating area, because our detection technique is very sensitive to the magnetization precession around the notch (a significant voltage drop across the sample occurs in this region).

(ii) At zero field [Fig. 5(b.2)] the area in which the magnetization is found to oscillate at $3 \mathrm{GHz}$ is only a tiny region, in good agreement with the absence of a distinguishable feature in our measurements. It is interesting to note here that the vortex wall does not move at this frequency since vortex wall motion is usually observed well below $1 \mathrm{GHz}$.

(iii) Around $-10 \mathrm{mT}$ [Figs. 5(c) and 5(d)] we clearly observe the depinning of the DW when the force exerted by the magnetic field exceeds the pinning force of the notch. The region that is found to oscillate just before the switching field is the inhomogeneous part of the DW but also the domains on both sides. Nevertheless we think that the peak we observe in the rectified voltage comes mainly from the DW part. This is due to the increased sensitivity of our detection in the notch area where current lines are denser and the fact that our gold contacts select a reduced region as represented by the red lines in Fig. 5. This is also supported by the fact that the two peaks we measure-the nucleation peak, due to the DW oscillation, and the switching peak-have comparable heights. Indeed the latter peak would be much higher if the domain contribution were dominant.

(iv) Finally, around $-33 \mathrm{mT}$, the magnetic configuration is not fully saturated but the field is high enough to remove the closure domains at both ends of the stripe and the region that oscillates is the area around the notch vertex.

It is possible to use these simulations more quantitatively (along with the simulations of the current lines) in order to estimate the AMR changes expected. The AMR contribution due to magnetization precession at $3 \mathrm{GHz}$ when sweeping the static field at angle $\theta$ of 90 degrees is shown in Fig. 6. We find that there is a good quantitative agreement with the data

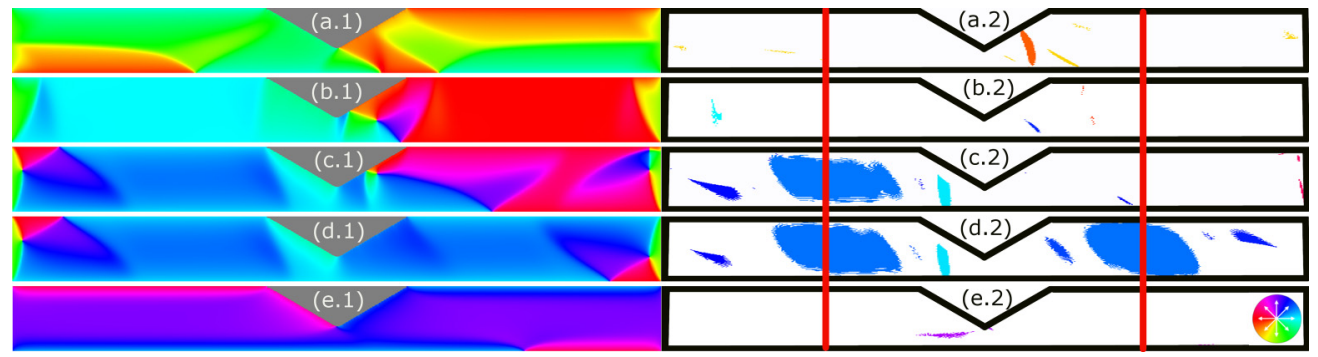

FIG. 5. (Color online) (x.1) Static magnetization configuration as found during the simulation of the experimental field scan (when sweeping the field downwards from positive transverse saturation) using MuMax2. (x.2) In color are the areas in which the magnetization oscillates when a $3 \mathrm{GHz}$ AC field is superimposed at five different DC magnetic field values: $10 \mathrm{mT}$ (a), $0 \mathrm{mT}$ (b), $-10 \mathrm{mT}$ (c), $-10.5 \mathrm{mT}$ (d), and $-32.5 \mathrm{mT}$ (e). The color code used for the in-plane magnetization orientation is shown in the inset. Bold red lines on the right indicate the position of the voltage contacts on the stripe. 


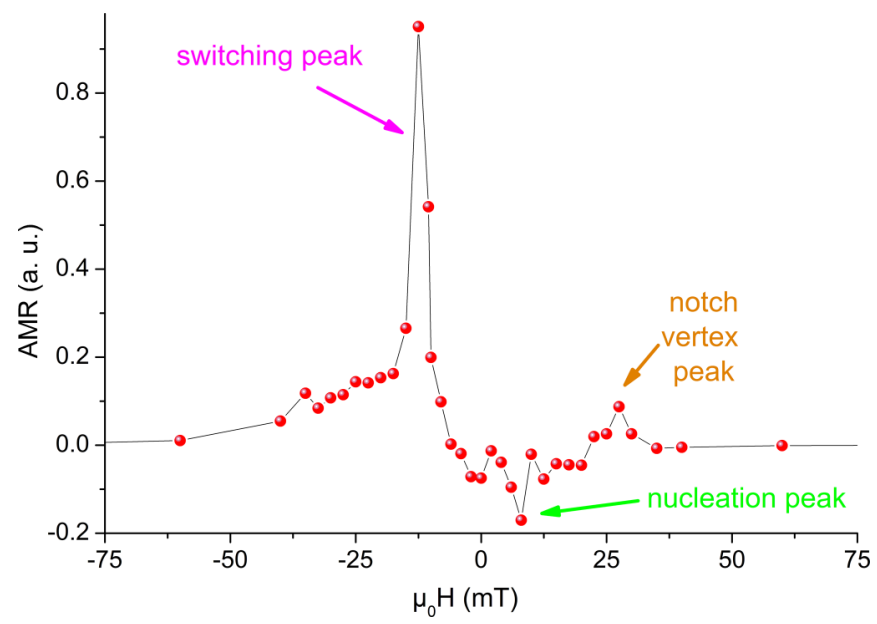

FIG. 6. (Color online) AMR contribution obtained from the micromagnetic simulations due to magnetization precession at $3 \mathrm{GHz}$ when sweeping the static field at an angle $\theta$ of 90 degrees.

including in the peak positions, which proves the accuracy of these simulations.

It is interesting to go further with the simulations and explore the response at higher frequencies for which we do not measure any particular feature in the rectified voltages. We show in Fig. 7 the areas in which the magnetization is found to oscillate at different frequencies below and above $6 \mathrm{GHz}$. As can be seen in this figure, at any frequency below $6 \mathrm{GHz}$, some region around the DW can resonate, but nothing happens above. This again reinforces our conclusion that the largely inhomogeneous internal field of the DW provides regions matching the resonance condition for any frequency below a cutoff found to be $6 \mathrm{GHz}$ in our system.

Now we turn our attention to the influence of a dc current on these DW resonances. For this aim we inject different dc currents into the sample while measuring the rectified voltage in the same conditions as described above. The field dependence of the rectified voltage measured at $3 \mathrm{GHz}$ while applying different dc current values up to $10^{6} \mathrm{~A} \mathrm{~cm}^{-2}$ for a magnetization at an angle of 85 degrees from the stripe axis is displayed in Fig. 8. The voltage signals are noisier but still clear and significant. As the current density increases above

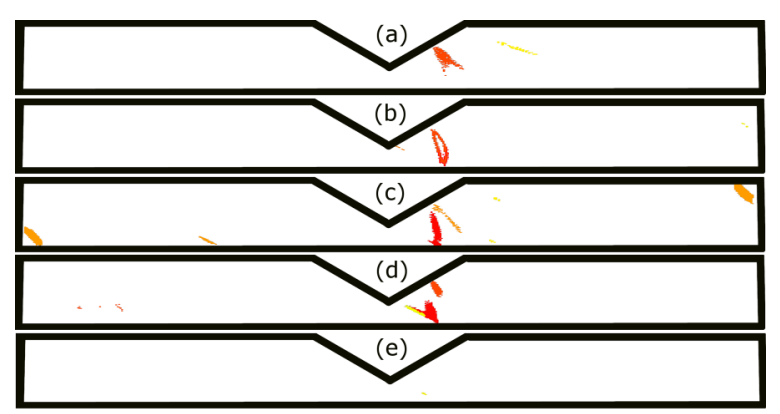

FIG. 7. (Color online) In color are the areas in which the magnetization oscillates at $100 \mathrm{Oe}$ at different frequencies: $1.71 \mathrm{GHz}$ (a), $2.40 \mathrm{GHz}$ (b), $4.00 \mathrm{GHz}$ (c), $4.86 \mathrm{GHz}$ (d), and $7.40 \mathrm{GHz}$ (e). The color code used for the in-plane magnetization orientation is shown in the inset of Fig. 5.

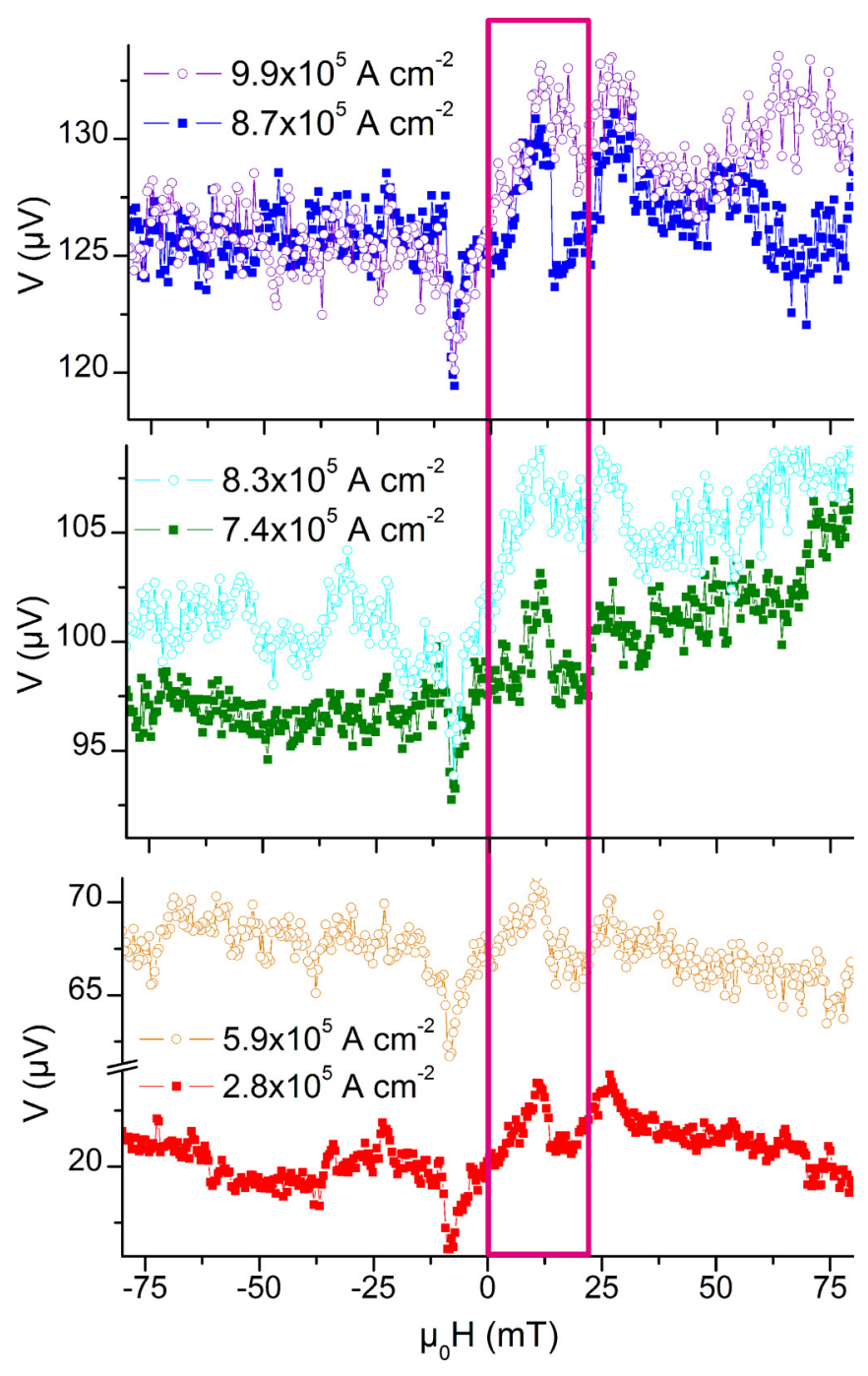

FIG. 8. (Color online) Field dependence of the measured rectified voltage when applying different dc current densities to the Py stripe at $3 \mathrm{GHz}$ when the angle between the static magnetic field and the stripe axis is 85 degrees.

$8 \times 10^{5} \mathrm{~A} \mathrm{~cm}^{-2}$, the nucleation peak broadens demonstrating that spin torque starts to play a role on the DW FMR profiles at current densities two orders of magnitude lower than the depinning ones. A quantitative description of this effect is beyond the scope of the present paper. However, the sensitivity of the rectified voltage measurement allows us to conclude that the domain wall behavior is modified at rather low current densities. This is consistent with models predicting that the internal structure of domain walls should start to distort largely before depinning [18-20].

\section{CONCLUSIONS}

In summary, we have demonstrated here that internal DW resonances can be detected by an electrical technique. The measurement is based on the voltage signal generated by rectification of the induced current coupled to the AMR oscillation. 
This rectification technique is extremely sensitive as it allows us to measure signals from very localized precessing regions within a single pinned DW. Micromagnetic simulations, performed using the freely available code MuMax2, have enabled a full understanding of the measured voltage signal and the proper identification of the different resonances we observe.

Our measurements, along with the micromagnetic simulations, demonstrate that DWs provide a sufficiently varying internal field so that some resonance can always occur below a cutoff frequency of $6 \mathrm{GHz}$. Lastly, we showed that the
DW resonances are affected by a DC current two orders of magnitude below the depinning threshold.

\section{ACKNOWLEDGMENTS}

We would like to acknowledge financial support from the European Commission through the Marie Curie Intra European Fellowship Project No. 301656: AtomicFMR, funded by the 7th Framework Programme. We also would like to thank J. V. Kim for his kind help with MuMax2 simulations and S. Rohart for assistance with Py deposition.
[1] S. A. Wolf, D. D. Awschalom, R. A. Buhrman, J. M. Daughton, S. von Molnar, M. L. Roukes, A. Y. Chtchelkanova, and D. M. Treger, Science 294, 1488 (2001).

[2] I. Zutic, J. Fabian, and S. Das Sarma, Rev. Mod. Phys. 76, 323 (2004).

[3] N. Mecking, Y. S. Gui, and C. M. Hu, Phys. Rev. B 76, 224430 (2007).

[4] M. Hayashi, L. Thomas, C. Rettner, R. Moriya, and S. P. Parkin, Nat. Phys. 3, 21 (2007).

[5] O. Rousseau and M. Viret, Phys. Rev. B 85, 144413 (2012).

[6] L. Berger, J. Appl. Phys. 55, 1954 (1984).

[7] L. Berger, Phys. Rev. B 73, 014407 (2006).

[8] D. Bedau, M. Klaui, M. T. Hua, S. Krzyk, U. Rudiger, G. Faini, and L. Vila, Phys. Rev. Lett. 101, 256602 (2008).

[9] E. Saitoh, H. Miyajima, T. Yamaoka, and G. Tatara, Nature (London) 432, 203 (2004).

[10] H. J. Juretschke, J. Appl. Phys. 31, 1401 (1960).
[11] Y. S. Gui, N. Mecking, and C. M. Hu, Phys. Rev. Lett. 98, 217603 (2007).

[12] M. V. Costache, S. M. Watts, M. Sladkov, C. H. van der Wal, and B. J. van Wees, Appl. Phys. Lett. 89, 232115 (2006).

[13] A. Yamaguchi, H. Miyajima, T. Ono, Y. Suzuki, S. Yuasa, A. Tulapurkar, and Y. Nakatani, Appl. Phys. Lett. 90, 182507 (2007).

[14] A. Ben Hamida, O. Rousseau, S. Petit-Watelot, and M. Viret, Europhys. Lett. 94, 27002 (2011).

[15] O. Rousseau, S. Petit-Watelot, and M. Viret, J. Phys.: Condens. Matter 24, 024211 (2012).

[16] J. Grollier, M. V. Costache, C. H. van der Wal, and B. J. van Wees, J. Appl. Phys. 100, 024316 (2006).

[17] A. Vansteenkiste and B. Van de Wiele, J. Magn. Magn. Mater. 323, 2585 (2011).

[18] J. He, W. Li, and S. Zhang, J. Appl. Phys. 98, 016108 (2005).

[19] X. Waintal and M. Viret, Europhys. Lett. 65, 427 (2004).

[20] A. Vanhaverbeke and M. Viret, Phys. Rev. B 75, 024411 (2007). 